\title{
Uma análise de aspectos relacionados ao desenvolvimento e adoção de Enterprise Resources Planning livre de código aberto
}

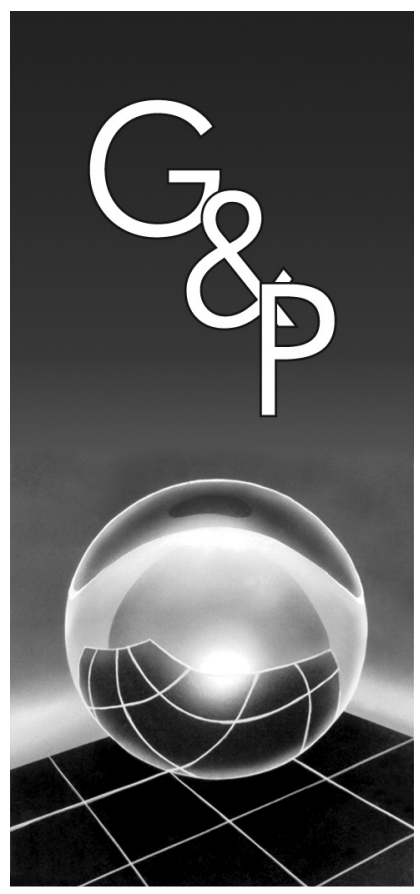

\author{
Rogério Atem de Carvalho \\ Renato de Campos
}

\begin{abstract}
Resumo
Sistemas Integrados de Gestão ou Enterprise Resources Planning - ERP possibilitam o processamento das informações necessárias em uma empresa usando um único banco de dados. Muito tem se escrito sobre este tipo de software, abordando questões como o alto custo da aquisição de licenças, e a dependência de consultoria para a sua adaptação e implantação nas empresas. Atualmente vem crescendo o desenvolvimento e uso de ERP Livre de Código Aberto (FOS-ERP). Porém verifica-se que este tipo de sistema ainda não é suficientemente explorado, mesmo no meio acadêmico. Este artigo relata alguns trabalhos publicados sobre o assunto e levanta questões que devem ser tratadas por pesquisadores e demais interessados para adequar e viabilizar o uso desses sistemas conforme a realidade nacional. Assim, após uma introdução ao tema, são apresentadas algumas diferenças entre o FOS-ERP e seus equivalentes proprietários (Proprietary ERP ou P-ERP) em termos de modelos de negócios, seleção, customização e evolução. Em seguida são elencados alguns desafios e oportunidades que o FOS-ERP pode oferecer a usuários, fornecedores, pesquisadores e colaboradores individuais. Concluindo, este artigo busca ampliar a discussão sobre FOS-ERP, destacando fatores tais como seu potencial de inovação tecnológica e estratégias de negócios.
\end{abstract}

Palavras-chaves: Sistemas integrados de gestão. ERP. Software livre. Código aberto. Desenvolvimento de software. Estratégias de negócios.

\section{Introdução}

É de reconhecida importância a necessidade de integração no processamento de informações das empresas. Atualmente Sistemas Integrados de Gestão (também conhecidos como Enterprise Resources Planning - ERP) fornecem suporte aos processos de negócios integrando os dados da empresa em um único banco de dados. A sua comercialização normalmente é feita na forma de pacotes de software, cujos módulos podem ser implantados de acordo com as necessidades dos clientes. O investimento no processo de implantação é elevado e o resultado nem sempre é o esperado (GONÇALVES et al., 2004).

Um ERP pode ser implementado como ele é originalmente (padrão), porém, um nível de adaptação é necessário. O processo de adaptação de um ERP pode ser compreendido por parametrização e/ou customização, sendo de fundamental importância na implantação do mesmo (TIJUNELIS; BARRELLA, 2003). Segundo Hong e Kim (2002), o processo de adaptação do ERP é dividido em três níveis: configuração ou customização, extensão e modificação. A configuração trata de preencher as tabelas do sistema com os parâmetros da empresa sem alterar o código fonte, a extensão visa o desenvolvimento de aplicativos em linguagem específica, que serão ligados ao ERP, e a modificação altera o código fonte ou núcleo do produto.

Mendes e Escrivão Filho (2007) propõem um roteiro para adoção de sistemas ERP para pequenas e médias empresas (PME), no qual se percebe a preocupação com a adequação:

- Avaliação da necessidade de ERP (analise de possíveis problemas e soluções da empresa);

- Seleção e adequação (análise e verificação da adequação de funcionalidades do sistema);

- Implantação (planejamento das atividades que precedem a implantação até a consumação da mesma, envolvendo parametrização e customizações previstas na fase anterior);

- Conscientização e treinamento (realização de palestras, seminários e treinamentos); e 
- Utilização (uso e modificações do sistema para atender a mudanças nas regras de negócio).

Para Jesus e Oliveira (2007), adotar um ERP não implica somente na transformação de uma organização tradicional em uma organização integrada, mas são necessárias mudanças complexas que abrangem aspectos estruturais e comportamentais.

Conforme Silva e Pereira (2006), a adaptação dos ERPs na empresa pode ser facilitada pela adoção de ferramentas e metodologias de implementação que suportem desde a identificação das necessidades do cliente até adaptação de sistemas complexos ao negócio. Ainda segundo os autores, tanto o fornecedor como os clientes acabam enfrentando problemas para a implementação de um ERP.

Quando empresas usuárias compram licenças de ERP da empresa que os desenvolveu e tem a propriedade do software, neste trabalho chamado de ERP Proprietário ou $P$-ERP (Proprietary ERP), muitas vezes os usuários ficam dependentes da consultoria do desenvolvedor e limitados com relação a possíveis adaptações do código do software.

Nos últimos anos, surgiram sistemas classificados como ERP Livres de Código Aberto (FOS-ERP, do inglês Free Open Source - ERP).

A Free Software Foundation-FSF define como "software livre" aquele que dá ao usuário a liberdade de executar o programa para qualquer propósito, estudar como o programa funciona, adaptá-lo às suas necessidades, redistribuir cópias, melhorar o programa e liberar as suas melhorias ao público, de modo que toda a sua comunidade se beneficie delas.

De acordo com a Open Source Initiative - OSI, as licenças de software consideradas de "código aberto" devem atender a dez condições:

1. O software pode ser livremente distribuído ou vendido;

2. O código fonte deve estar incluído ou disponível livremente;

3. Redistribuição de modificações deve ser permitida;

4. Licenças podem requerer que modificações sejam distribuídas em separado com opção de adoção;

5. Não pode haver discriminação de pessoas ou grupos;

6. Não pode haver discriminação contra esforços de desenvolvimento;

7. Os direitos associados ao programa devem se aplicar a todos aos quais o programa é distribuído, sem a necessidade da obtenção de licenças adicionais por estes;

8. O programa não pode ser licenciado como parte de uma distribuição maior;

9. A licença não pode forçar que outros softwares distribuídos juntos sejam de código aberto também; e

10. A licença deve ser independente da tecnologia.

Os sistemas ERP Livres de Código Aberto (FOS-ERP) estão desenvolvendo aceitação crescente e, consequentemente, melhorando sua participação no mercado. De acordo com um estudo de mercado da International Data Corporation - IDC, os serviços relacionados a FOS-ERP somariam cerca de US\$ 36 bilhões no final de 2008 (LECLAIRE, 2006). Kissinger (2008) destaca que, embora dados de mercado sobre FOS-ERP sejam difíceis de obter, tem havido um crescimento contínuo, com "cerca de vinte fornecedores realizando pelo menos U\$100 milhões anuais de faturamento".

As razões para este fenômeno são basicamente duas: custos menores e livre acesso ao código do aplicativo. Assim, têm aparecido empresas que dão suporte a esta categoria de sistemas, tais como os sistemas Compiere, ERP5 e OpenBravo. Do ponto de vista do custo, em geral, o licenciamento desses sistemas implica em investimentos reduzidos ou mesmo nenhum investimento. Do ponto de vista do acesso ao código, permanece a percepção de, se a customização é inevitável, por que não adotar uma solução que exponha o seu código à empresa cliente, a qual poderia adaptar livremente o sistema às suas necessidades?

Considerando o aumento nas implantações do FOS-ERP e o número relativamente pequeno de referências a este assunto na literatura, este artigo se propõe apresentar tendências que influenciam diretamente o segmento FOS-ERP, destacar as diferenças entre o FOS-ERP e o ERP Proprietário $(P-E R P)$ em termos de modelos de negócios, seleção, customização e manutenção e identificar os desafios e as oportunidades que eles oferecem a stakeholders e comunidades de desenvolvedores.

\section{Alguns estudos e discussões sobre 0 Free Open Source Enterprise Resources Planning}

Esta seção pretende abordar alguns estudos iniciais sobre FOS-ERP, os quais apresentam diferentes abordagens, tais como possíveis estratégias de negócio, formas de avaliação, metodologias e comunidades de desenvolvimento.

\subsection{Primeiros trabalhos e eventos relacionados ao tema}

Enquanto sua importância mercadológica aumenta, o FOS-ERP é ainda insuficientemente analisado pela academia, onde grande número de artigos concentra seus esforços na implantação do $P$-ERP, gerenciamento de projetos e aspectos econômicos (BOTTA-GENOULAZ et al., 2005). Uma série de aspectos relevantes que o diferencia do $P$-ERP não é ainda plenamente compreendida. Por exemplo, Carvalho (2006) mostra como a avaliação de FOS-ERP traz mais preocupações do que a avaliação de $P$-ERP. Na verdade existem muitos tópicos a serem explorados e tendências a serem confirmadas, já que o número de usuários e os períodos de adoção são ainda pequenos em relação às mesmas estatísticas para $P$-ERP. De acordo com Kim e Boldyreff (2005, p. 5), 
até setembro de 2005 somente um artigo sobre o ERP de Código Aberto (SMETS-SOLANES; CARVALHO, 2003) tinha sido publicado, considerando todos os periódicos e eventos da Association for Computing Machinery - ACM e da IEEE Computer Society, enquanto que um grande número de artigos fora publicado nas revistas comerciais não-acadêmicas.

Por exemplo, Carvalho (2006) foi o primeiro artigo sobre a avaliação de FOS-ERP. Em 2006 foi realizado o primeiro evento internacional sobre Sistemas de Informação Empresarial (Enterprise Information Systems - EIS) e FOS-ERP em Viena (First International Workshop on Freel Open Source EIS/ERP). Este evento foi realizado em conjunto com a International Conference on Research and Practical Issues of Enterprise Information Systems - CONFENIS, organizada pelo IFIP Working Group on EIS.

\subsection{Inovação e estratégia de negócios}

De acordo com Caulliraux et al. (2000), o ERP é estratégico, considerando-se que envolve um investimento significativo, com implicações, que não se restringem aoa financeiro. Eles são importantes também na formação de bens intangíveis, incluindo o autoconhecimento da empresa.

Apesar de não haver muitos trabalhos especificamente sobre FOS-ERP, alguns bons trabalhos sobre tópicos relacionados podem ser encontrados. Atualmente, a análise mais aprofundada sobre o impacto econômico do Software Livre e de Código Aberto (Free Open Source Software FOSS) em sistemas de gestão empresariais foi conduzida por Dreiling et al. (2005, p. 2), os quais argumentam que

\footnotetext{
"padrões, que supostamente são abertos e permitem o desenvolvimento e interoperabilidade de sistemas, tendem a ser influenciados e definidos pelos atores globais de sistemas empresariais de acordo com seus interesses".
}

Segundo os mesmos autores, a imposição dos interesses dos atores globais (como as grandes empresas de desenvolvimento de sistemas, que têm grande poder) têm como grave consequência a possível incompatibilidade com interesses da indústria de software como um todo, o que pode gerar efeitos sobre as economias locais e nacionais que não os tenham contemplado nesses padrões. E mais:

\begin{abstract}
"apesar do controle de interfaces e padrões por poucos desenvolvedores de software, até mesmo a integração da infraestrutura de informações de uma única empresa com uma marca de sistema empresarial pode não ser consolidada ao longo do tempo". (DREILING et al., 2005, p. 2)
\end{abstract}

Concluindo sobre padrões abertos, eles entendem que os princípios de engenharia de software e padrões abertos são necessários, mas não são uma condição suficiente para que o desenvolvimento do software das empresas se torne menos restrito pelas políticas dos atores globais, atenda aos interesses dos usuários e garanta uma indústria de software saudável em mercados regionais.

Do ponto de vista da inovação, Dreiling et al. (2005) declaram que muitos economistas concordam com a questão de que as empresas dominantes - como os atores globais de $E R P$-, os monopólios e os oligopólios estão menos dispostos a responder aos requisitos dos clientes - o que tende a restringir a inovação de produtos e serviços. Declaram também que o controle de arquiteturas por meio de software proprietário e padrões abertos na indústria de aplicativos empresariais dificulta a inovação, que poderia ser benéfica para vários usuários dos sistemas empresariais, principalmente nas economias menos desenvolvidas. Isto parece ser um problema sério, já que a adaptação é um ponto crucial na implantação do $E R P$, o qual deve ser adequado às necessidades específicas de cada empresa usuária. Porém, os atores globais "estão menos dispostos a responder aos requisitos dos clientes". (DREILING et al., 2005, p. 4).

Esta conclusão reforça uma consequência positiva da liberdade de manipulação do código do FOS-ERP pelo próprio adotante: se o fornecedor altera os termos do contrato, a empresa do cliente não está presa a apenas um fornecedor com uma solução específica e pode mudar para outra empresa que forneça o serviço de suporte (KOOCH, 2004). Além disso, duas empresas concorrentes podem gerar um diferencial estratégico utilizando o mesmo $E R P$. Embora este problema possa também acontecer com o FOS-ERP, parece ser de maior extensão para o $P$-ERP, já que, devido ao rígido controle sobre o código, as adaptações são limitadas à parametrização ou às alterações na funcionalidade, por meio de linguagens específicas e proprietárias, que restringem a diferenciação real e aumentam os custos de customização (CARVALHO, 2006). Portanto, se a integração entre os processos pode, por si mesma, tornar-se uma fonte de vantagem competitiva (CAULLIRAUX et al., 2000), isto pode ser extrapolado pela possibilidade de se alterar o código aberto, o que pode conduzir a uma vantagem ainda melhor.

Se, por um lado, o FOS-ERP pode fomentar a inovação e fornecer mais poder aos usuários que o adotam, por outro lado algumas perguntas precisam ser respondidas, considerando que este tipo de FOSS é ainda recém chegado no cenário de sistemas de gestão empresarial. Mesmo alguns entusiastas reconhecem que o nível de serviço dos fornecedores de FOS-ERP tem muito que melhorar e adquirir experiência, enquanto que, em contraste, o $P$-ERP conta com uma rede madura de consultores e um longo histórico de sucessos e fracassos (SERRANO; SARRIERI, 2006).

Xu (2003) propõe uma classificação de acordo com o posicionamento estratégico do usuário em relação a um FOSS, que pode ser associada a uma possível estratégia por parte de um adotante para com o FOS-ERP: 
- Consumer: quando o adotante tem um papel passivo, apenas usa o software, sem ter a intenção ou a habilidade de modificar ou distribuir os códigos;

- Prosumer: quando o adotante tem um papel ativo, relatando erros no software e apresentando pedidos de novas características, requisitos e dúvidas, e eventualmente fornecendo correções de erros, melhorias e novas funcionalidades;

- Profitor: neste caso existe um papel passivo em termos de desenvolvimento de software, porém este adotante usa o software como uma fonte de lucros (sem modificá-lo por si próprio); e

- Partner: neste último caso, o adotante tem um papel ativo, participando ativamente de todo o processo de desenvolvimento com o propósito de ter lucros.

\subsection{Métodos de avaliação para adoção de Free Open Source Enterprise Resources Planning}

Considerando as oportunidades e a avaliação de riscos dada a natureza estratégica da adoção de um ERP, Carvalho (2006) propõe um método de avaliação de FOS-ERP composto das seguintes fases: Preparar o processo de avaliação; Identificar as alternativas; Classificar os atributos da alternativa; Comparar as alternativas e Selecionar aquela que melhor se adapta às necessidades do adotante. Durante a fase de preparação, o adotante deve optar pelo comportamento de simples consumer - conforme classificação de Xu (2003) apresentada na seção anterior -, apenas obtendo a solução do fornecedor. Ou se tornar um prosumer, ao procurar integrar o sistema em si com outros software customizados ou desenvolvidos internamente. Esta é uma suposição genérica, já que na prática o vendedor pode impor condições específicas de licenciamento, as quais mantêm o software aberto mas restringem a implantação em condições que mantêm o controle do vendedor sobre ele. Logicamente, este tipo de posicionamento não é uma decisão trivial, já que envolve uma série de exigências, tais como conhecimento da plataforma e arquitetura do FOS-ERP, aprender a lidar com a comunidade de desenvolvimento - o que pode significar gerenciar exigências de diferentes stakeholders (WEST; O'MAHONY, 2005) e alocar recursos para o desenvolvimento. É importante pesar os ganhos diretos e indiretos de desenvolver partes do sistema com as desvantagens de se realizar tal ato.

Também, no sentido de propor uma sistemática para avaliação de um FOS-ERP, Herzog (2006) apresenta uma abordagem bastante abrangente, na qual identifica três métodos diferentes para a implementação de soluções baseadas em FOS-ERP (Selecionar um pacote; Desenvolver individualmente módulos necessários e não existentes no $E R P$; e Integrar a melhor das soluções) e cinco critérios para avaliação das alternativas (ajuste funcional, flexibilidade, suporte, continuidade e maturidade). Este método apresenta uma possibilidade interessante, ainda não muito bem explorada na prática: a de integrar soluções de diferentes fornecedores através das técnicas de Enterprise Application Integration - EAI (Integração de Aplicativos Empresariais). Um estudo de caso bem sucedido sobre integração de soluções de $P$-ERP é descrito por Alshawi et al. (2004), mas faltam exemplos na literatura sobre como realizar o mesmo com o FOS-ERP.

\subsection{Metodologias, linguagens e ferramentas de desenvolvimento}

Campos e Carvalho (2006), assim como Silva et al. (2006), relatam que são necessárias metodologias adequadas, linguagens e ferramentas de suporte para o desenvolvimento de sistemas tais como o FOS-ERP. Para que haja compatibilidade entre essas metodologias, linguagens e ferramentas, propõem-se o uso de Arquiteturas e Metodologias de Referência tais como Computer Integrated Manufacturing Open System Architecture - CIMOSA ou Generalised Enterprise Reference Architecture and Methodology-GERAM. GERAM reúne os conceitos genéricos de empresas recomendados para uso em projetos de integração e engenharia de sistemas de empresas. Ela fornece uma estrutura de análise e modelagem baseada no conceito de ciclo de vida e identifica três dimensões para definir o escopo e o conteúdo da modelagem de empresa representados na Figura 1 (IFIP-IFAC, 1999):

- Dimensão de ciclo de vida: dirige o processo de modelagem de entidades de empresa de acordo com as atividades do ciclo de vida, desde a fase de Identificação das entidades da empresa até a sua desativação;

- Dimensão de instanciação: controla o processo de particularização de modelos, do genérico para o

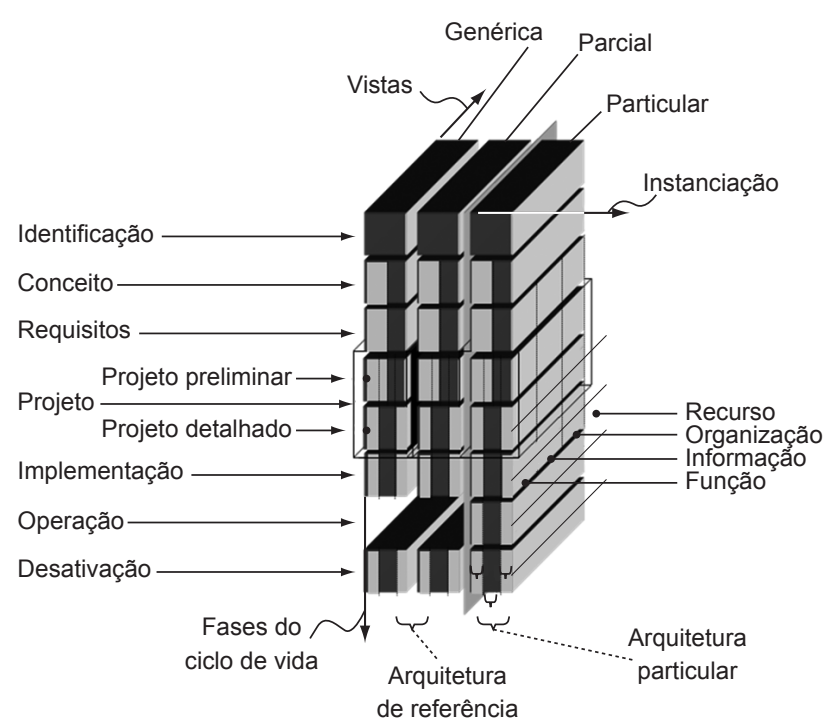

Figura 1. Estrutura de modelagem da arquitetura GERAM. Fonte: Adaptado de IFIP-IFAC, 1999. 
parcial (arquitetura de referência) e depois então para o modelo particular; e

- Dimensão de vistas: controla as vistas específicas da entidade de empresa, conforme os aspectos de Função, Informação, Organização e Recursos.

GERAM define sete fases de um ciclo de vida padrão que pode ser adaptado para qualquer entidade pertinente a uma empresa, assim como para todo o desenvolvimento e implantação de um ERP. Todas as fases estão apresentadas na Figura 1. Observa-se que a fase de Projeto é subdividida nas subfases Projeto Preliminar e Projeto Detalhado. Elas podem ser resumidas conforme descrição a seguir:

- Identificação: identifica especificamente a entidade a ser considerada, em termos de seu domínio e ambiente;

- Conceito: define a missão, visão, valores, estratégias e objetivos da entidade;

- Requisitos: envolve um conjunto de aspectos humanos, aspectos do processo e aspectos orientados pela tecnologia, assim como atividades necessárias para descrever os requisitos operacionais da empresa;

- Projeto: modela a entidade e auxilia na compreensão das funcionalidades do sistema;

- Implementação: o projeto é transformado em componentes reais e, após testes e aprovação, a operação do sistema é iniciada;

- Operação: é o uso real do sistema, incluindo o feedback por parte do usuário, o que pode conduzir a um novo ciclo de vida para a entidade; e

- Desativação: representa o desmonte da entidade, após o seu uso bem sucedido.

Silva et al. (2006) consideram a possibilidade de se adaptar o Processo Unificado (Unified Process - UP) como metodologia e de se usar a Unified Modeling Language - UML como linguagem de modelagem e desenvolvimento de um FOS-ERP. Carvalho e Campos (2006) e Monnerat et al. (2008) apresentam alternativas para o processo de desenvolvimento um FOS-ERP, com diferentes abordagens para as suas atividades, algumas mais relacionadas com a definição de requisitos de negócios, outras relacionadas com o desenvolvimento de códigos.

Rochetti (2009) e Galera et al. (2008) ressaltam a necessidade de se usar linguagens padrão, tal como a norma ISO 19440, para o levantamento de requisitos e para a documentação de modelos relacionados a um ERP. Eles entendem que essa questão é ainda mais importante para um FOS-ERP, pois se supõe que vários adotantes estarão lendo e trabalhando esses modelos para compreender, modificar e implementar os respectivos códigos. Usando uma linguagem de documentação padrão, pode-se até diminuir as barreiras no caso de comunicação entre membros de comunidades de desenvolvimento e adotantes que não possuem fluência em uma mesma língua estrangeira.
De qualquer forma, trabalhos acadêmicos devem "embutir" conceitos e boas práticas necessários ao bom desenvolvimento desses sistemas em ferramentas amigáveis, por exemplo, com funcionalidades para sistematizar procedimentos e transformar modelos de alto nível em código do software da maneira mais fácil, automática e consistente possível.

\subsection{Comunidades e empresas desenvolvedoras de Free Open Source Enterprise Resources Planning}

A fim de se compreender a dinâmica do FOS-ERP, assuntos que afetam os softwares abertos devem também ser abordados com uma visão ampla. Crowston e Howison (2006) e Carvalho et al. (2006) avaliam a saúde das comunidades de Código Aberto para verificar se um FOSS é adequado às necessidades daqueles que adotam ou contribuem com o software. Esse tipo de avaliação pode ser empregado como uma das ferramentas para verificar a maturidade de um FOS-ERP. Avaliar as comunidades do FOS-ERP significa compreender o comportamento de outras organizações relacionadas ao projeto, já que o $E R P$, em geral, não serve para uso individual, e os que geralmente mais contribuem com o projeto do sistema, na maioria das vezes, são empresas, e não pessoas. Assim, para entender as diferenças entre este e outros tipos de software aberto, é necessário conhecer como se comportam os projetos FOSS organizados pelas comunidades e patrocinados comercialmente. De acordo com West e O'Mahony (2005, p. 2), uma das questões críticas do FOSS comercial é a fase de iniciação: quando o código do projeto é aberto,

\section{"uma nova comunidade se apresenta a um grande e complexo sistema, o qual pode ser mais difícil de decifrar".}

Assim, o criador do FOS-ERP poderá ter de esperar até que as contribuições de outras empresas tornem-se viáveis e também vantajosas. O principal incentivo econômico para a participação da empresa é a emancipação das condições de preço e licença impostas por grandes empresas de software (WANG et al., 2005), mas o potencial para realizar tal substituição deverá estar apenas latente no início do projeto. O mesmo tipo de incentivo é identificado por Riehle (2007, p. 26), que diz que os provedores de soluções podem aproveitar o software de código aberto

\footnotetext{
"porque eles aumentam os lucros através de economia direta de custos e da habilidade de atingir mais clientes através da melhoria da flexibilidade em termos de preços relacionados ao sistema".
}

Outro aspecto com relação ao fornecedor identificado por estes autores é que a abertura do código pode reduzir os custos do teste do software e as tarefas de Pesquisa e Desenvolvimento. Estas vantagens estão estimulando uma melhor aceitação de mercado, de acordo com o 
estudo de Zinnov (2006), o qual, entre outras coisas, mostra números sobre o aumento da participação do capitalismo de risco e a maior penetração no mercado dos EUA de sistemas de gestão empresarial de código aberto. Além disso, Goth (2005) afirma que o mercado de software aberto é uma "segunda onda" com relação ao software empresarial e que os modelos empresariais de FOSS estão finalmente prontos para enfrentar este novo desafio de mercado. Estas duas últimas referências indicam uma melhoria geral do relacionamento entre as comunidades de FOSS e os usuários dos sistemas de gestão empresariais.

Apesar dessas diferenças, o FOS-ERP e o P-ERP certamente possuem uma coisa em comum: ambos têm uma empresa por trás de suas atividades de implantação. Embora existam FOS-ERP mantidos quase que somente por comunidades formadas basicamente por indivíduos, como o GNU Enterprise, parece que somente os FOS-ERP patrocinados por empresas, tais como Compiere, ERP5 e OpenBravo são os bem sucedidos. Em outras palavras, os FOS-ERP são normalmente do tipo código aberto comercial, no qual "uma entidade que visa lucro opera e desenvolve" o sistema, de acordo com a classificação de Riehle (2007, p. 25).

\section{Diferenças entre Free Open Source Enterprise Resources Planning e Proprietary Enterprise Resources Planning}

A exposição do código do FOS-ERP força fornecedor e adotante a conviverem com o fato de que a customização e a manutenção podem ser realizadas por outra empresa ou mesmo pelo usuário, em vez do fornecedor original. Isto significa que o adotante está livre para escolher o nível de participação do fornecedor nas diferentes fases do ciclo de vida do $E R P$, sendo que, até certo ponto, a participação do fornecedor também pode ser customizada. Analisar as diferenças entre o ERP de código aberto e o proprietário depende do lado da relação comercial em que a organização está: adotante (usuário) ou fornecedor.

\subsection{Diferenças para o adotante}

Selecionar um ERP para adoção é um projeto complexo porque, além da extensão da tarefa, este é um componente que exerce um grande impacto na organização adotante em termos financeiros e de autoconhecimento. Portanto, é importante utilizar uma abordagem metodológica para compreender adequadamente como as características do código aberto podem impactar neste tipo de projeto. Nesse sentido podem-se usar as fases do ciclo de vida da arquitetura GERAM, conforme já descritas na seção 2.4 (Figura 1). Exceto pelas fases identificação e desativação, as quais não são influenciadas pelos modelos de licenciamento, as demais fases podem ser utilizadas para uma melhor compreensão de como o FOS-ERP difere do P-ERP. Baseado nessas fases, a seguir são descritos aspectos-chave para a avaliação de alternativas e o refinamento sucessivo dos objetivos, requisitos e modelos relacionados à implementação desses sistemas.

\subsubsection{Fase de conceito}

Durante esta fase são estabelecidos objetivos de alto nível, tais como estratégia de aquisição, estimativas preliminares de tempo e custos e o impacto esperado com a adoção do $E R P$. No caso do FOS-ERP, o nível de envolvimento do adotante no desenvolvimento pode ser estabelecido. Em outras palavras, neste ponto o adotante pode começar a considerar a possibilidade de contribuir ativamente para um projeto de código aberto, tornando-se um prosumer. Logicamente que esta decisão final será possível somente durante as fases mais avançadas, quando o adotante conhecer melhor os requisitos e as alternativas.

\subsubsection{Fase de requisitos e fase de projeto preliminar}

Considerando o fato de ser a maior parte do desenvolvimento e customização de software hoje em dia feita através de ciclos de vida interativos e incrementais, pode-se dizer que não há limites claros entre os requisitos e as fases de projeto preliminar e entre o projeto detalhado e as fases de implementação. Desta forma, nesta análise essas fases são consideradas em conjunto.

A fase dos requisitos trata dos requisitos funcionais e não funcionais do sistema. $\mathrm{O}$ adotante poderá modelar alguns dos principais processos de negócios - parte do projeto preliminar - como uma forma de verificar como as alternativas se ajustam a eles. Neste ponto, o FOS-ERP começa a se diferenciar mais do $P$-ERP. A avaliação do $P$-ERP envolve a comparação de alternativas à luz da funcionalidade, o Custo Total de Propriedade (Total Cost of Ownership - TCO) e critérios tecnológicos. Para o FOS-ERP estes critérios, e outros relacionados especificamente ao FOSS, devem ser igualmente levados em consideração - lembrando que, embora sua implementação possa representar um impacto financeiro menor, em termos de autoconhecimento da empresa ela pode assumir uma importância bem maior, já que não só sustenta um inventário de registros e procedimentos, como também tais registros e procedimentos são percebidos de forma tecnológica - através do código aberto.

Em outras palavras, um FOS-ERP pode apresentar um impacto financeiro menor, mas na inovação e no conhecimento o impacto pode ser bem maior. Embora o $P$-ERP seja também altamente parametrizado, e adaptável através de linguagens dedicadas de programação, o acesso ao código aberto no FOS-ERP pode levar a uma exploração bem melhor das capacidades do ERP $e$, desta forma, permitir uma melhor implementação de soluções diferenciadas. 
Deste ponto de vista, o posicionamento estratégico de um adotante em relação a um FOS-ERP parece ser de máxima importância, dada a possibilidade de originar uma vantagem competitiva a partir do código aberto. Portanto, o adotante deve optar pelo comportamento de simples consumer, apenas obter a solução do fornecedor, ou se tornar um prosumer, ao integrar passivamente módulos de sistemas proprietários adquiridos a módulos de sistemas customizados ou ativamente desenvolvidos. Assim, fica claro que, quando um adotante considera o FOS-ERP como uma alternativa, ele deve também considerar desenvolver ou modificar partes dele a fim de adequá-lo a suas necessidades. Como foi visto anteriormente, este tipo de posicionamento envolve uma série de exigências de conhecimento tecnológico e de gestão do processo de desenvolvimento, com o envolvimento direto ou indireto de uma comunidade.

\subsubsection{Fase de projeto detalhado e fase de implementação}

A fase do projeto detalhado foca-se no refinamento de modelos e está associada à modelagem do processo de negócio e à identificação de parâmetros e definição de seus valores. A fase de implementação concentra-se na validação, integração de módulos e liberação para o uso inicial.

Se o adotante decidiu participar ativamente do projeto do FOS-ERP, decisões mais importantes sobre o projeto estarão envolvidas, tais como a criação de módulos inteiramente novos ou a extensão da estrutura básica. Uma consequência de assumir um papel mais ativo é o investimento em mais recursos humanos e financeiros para o aprendizado da plataforma e estrutura do FOS-ERP, desenvolvimento e realização da manutenção de suas partes, assim como administração do relacionamento com a comunidade do projeto. Nesse caso, os contratos de customização e manutenção devem definir as responsabilidades de cada parte no processo de implantação. Por exemplo, o que o fornecedor deve fazer se o adotante descobre uma falha no código que está em adaptação? Qual a prioridade que deve ser seguida pelo fornecedor a fim de corrigir esse defeito? $\mathrm{Na}$ verdade, é o fornecedor responsável pela correção desse defeito, já que para esta parte o adotante decidiu usar a licença livre da solução, desta forma isentando o fornecedor da responsabilidade pelo defeito?

Devido à possibilidade da abertura do código e de licenças flexíveis, o adotante possui ainda a opção de assumir níveis diferentes de envolvimento para cada fase. Para módulos comuns, como folha de pagamento, o adotante pode deixar o fornecedor realizar o trabalho. Entretanto, para os módulos estratégicos, onde o adotante acredita que mantém vantagem competitiva nos processos de negócio relacionados, ele pode desempenhar um papel ativo a partir do projeto detalhado de implementação e manutenção, para certificar-se de que as informações, ou pelo menos os detalhes mais estratégicos e que mantêm a vantagem competitiva serão preservados pelo fornecedor. Nesta situação, o fornecedor está limitado a agir como um tipo de consultor para o adotante. Pode-se achar possível manter segredo sobre partes do sistema ao contratar-se adequadamente um fornecedor de $P-E R P$ - o que em parte é verdade -, mas o adotante se tornará dependente do fornecedor em uma parte estratégica do sistema. Tornar-se dependente significa aguardar pelas prioridades do fornecedor ou pagar um alto preço para tornar-se prioridade quando mudanças forem necessárias. Mesmo se um adotante de $P$-ERP decidir desenvolver essas partes altamente estratégicas, ele terá que lidar com custos de licenciamento, de qualquer forma.

Um ponto muito interessante é a abertura das partes customizadas e patrocinadas por um adotante específico. Talvez este adotante não queira de forma alguma se tornar um desenvolvedor (o que é mais provável de acontecer), mas ele ainda quer manter algumas partes customizadas do sistema em segredo. Nesse caso, o fornecedor deve adaptar as licenças livres que ele adota para o seu ERP, de forma que a abertura geral do código esteja garantida, enquanto algumas partes customizadas e patrocinadas pelo cliente possam ser mantidas fechadas. Embora em termos de FOSS isto pareça um contrasenso, na realidade é uma situação comum no caso do FOS-ERP. Por exemplo, os autores conhecem um caso em que uma empresa adotante patrocinou todo o desenvolvimento de um FOS-ERP durante um período de três anos, sem se tornar um prosumer e mantendo apenas um algoritmo específico, relacionado à programação de preço dos produtos, em segredo. A licença original do FOS-ERP precisou ser alterada para se adequar às exigências deste cliente.

\subsubsection{Fase de operação}

Durante a fase de operação, os recursos da entidade (empresa) são gerenciados e controlados de forma a garantir a execução dos processos necessários para a mesma, a fim de cumprir a sua missão. Desvios das metas e objetivos ou feedbacks do ambiente podem levar às solicitações de mudança; portanto, durante esta fase ocorrem a manutenção e a evolução do sistema. Durante a operação, o adotante pode decidir, a qualquer momento - a não ser que haja impedimento devido a cláusulas contratuais -, mudar de fornecedor ou assumir ele mesmo a manutenção do sistema. Pequenas mudanças podem também ser conduzidas pelo próprio adotante ou mesmo pelos indivíduos da comunidade, os quais podem auxiliar em assuntos específicos.

Como uma observação conclusiva das diferenças do FOS-ERP do ponto de vista do adotante, a experiência tem mostrado que, na maioria das vezes, o adotante não se envolverá na customização e nem mesmo nas tarefas de manutenção. Ainda assim, o FOS-ERP pode ser uma boa escolha, já que ele reduz a dependência do fornecedor. Além disso, a abertura do código do FOS-ERP também torna 
mais fácil a sua adaptação para necessidades específicas, desta forma reduzindo os custos em customização e outras evoluções do software. Em outras palavras, os pontos centrais para se considerar são: redução do custo e liberdade de escolha.

Neste ponto cabe ressaltar o modelo novo de negócio em FOS-ERP que representa o ERP5 Express (http://www. erp5.com). Tecnologicamente esta versão do software é na forma de Software as a Service (SaaS), ou seja, a aplicação em si é executada remotamente e fica hospedada nas máquinas do fabricante do software ou de parceiros, configuração esta que não é novidade no mundo dos ERP. A inovação está no formato de negócio oferecido, que prevê a possibilidade de adoção incremental do software, seguindo um contínuo onde primeiro se adota o software como ele é, utilizando módulos básicos e na configuração SaaS; posteriormente, ainda como SaaS, são realizadas customizações em módulos estratégicos e, finalmente, o adotante pode "baixar" localmente (para sua infra estrutura de Tecnologia da Informação) o software customizado e todos os dados armazenados. Esse formato de adoção reduz substancialmente os riscos e os custos de adoção, permitindo que a organização usuária adote incrementalmente os processos de gestão integrada e evolua sua infra estrutura para absorver os mesmos. Se em qualquer momento o adotante desistir de empregar o ERP5, este possui mecanismos de exportação que permitirão recuperar os dados e os gabaritos dos processos de negócio (workflows) mantidos pelo sistema. Assim, o adotante mantém não só o controle sobre os dados, mas também sobre o código dos processos que os manipulam, bem como pode gerenciar melhor os riscos de implementação. Outro detalhe é que assinaturas de módulos básicos e sem customização começam em 15 Euros mensais, uma faixa de valor indubitavelmente muito baixa para o mundo ERP, mesmo para uma versão empacotada do software. Este modelo é muito recente ainda, tendo se iniciado em 2008, e oferecido apenas pelo ERP5, sendo assim serão necessários alguns anos para que seus efeitos sejam avaliados.

\subsection{Diferenças para o fornecedor}

Os modelos empresariais do fornecedor de FOS-ERP refletem a liberdade de escolha do cliente e das características mercadológicas gerais pelo fato de o código estar disponível. Como em outros tipos de FOSS, se, por um lado, os fornecedores beneficiam-se das melhorias implementadas e do trabalho de verificação feitos pela comunidade, por outro eles enfrentam a competição desta mesma comunidade ao lidar com a implantação e a manutenção. Na verdade, como mostrado anteriormente, até mesmo um adotante pode tornar-se, até certo ponto, um concorrente. É importante perceber que há três tipos de fornecedor: o criador do sistema original, os seus parceiros e os fornecedores free-lancer. No caso dos parceiros, um acordo formal e na maioria das vezes contratual é estabelecido entre eles e o criador do sistema. Este acordo envolve algumas responsabilidades para o parceiro, em especial, a conformidade às práticas de implantação do criador, a comunicação de novos negócios gerados pelo sistema, a abertura de código de partes novas ou melhoradas e o auxílio nas tarefas de desenvolvimento administradas pelo criador. Os free-lancers estão isentos destas obrigações, e, como consequência, não recebem nenhum tratamento especial por parte do criador, que espera que ao menos abram o código de melhorias implementadas por eles, seguindo o código de ética geral do FOSS. Na prática, este retorno dos free-lancers muitas vezes não ocorre. Seguindo o raciocínio comum sobre a definição de preços para o FOSS, os fornecedores do FOS-ERP podem aproveitar o software de código aberto porque, de acordo com Riehle (2007), este aumenta os lucros através de economia direta de custos e por meio da capacidade de atingir mais clientes através de uma melhor flexibilidade de preços, como mostrado na Figura 2. Ela mostra uma situação que talvez seja mais aplicável aos parceiros e free-lancers de FOS-ERP, que podem migrar do software proprietário, o qual demanda pagamento de licenças e assim arca com custos desse sofware proprietário (caso do $P$-ERP, Figura 2a), para os livres de código aberto com menores custos relacionados com o código do FOS-ERP (Figura 2b). Desta forma podem aumentar potencialmente a sua margem de lucro através de uma melhor combinação entre preço e quantidade de consumidores.

O criador deve saber como gerenciar a comunidade do projeto, descobrindo futuros parceiros, contratando - sempre que possível - pessoas que se tornem altamente produtivas no projeto e até mesmo tentando transformar os free-lancers em parceiros. Como seus companheiros proprietários, o FOS-ERP precisa de uma rede de parceiros que possa auxiliar na implantação de projetos onde o criador não possui condições de ser o contratante principal, além de descobrir novos mercados e clientes para o sistema. Contudo, unir colaboradores para iniciar um projeto de FOS-ERP pode ser uma tarefa difícil. Como dito anteriormente, os usuários de ERP são as organizações, não as pessoas, e, portanto, o criador deve aprender como atrair empresas parceiras que desejam voluntariamente contribuir sem tornarem-se concorrentes. Como conclusão principal, os fornecedores de FOS-ERP devem esforçar-se muito para formar uma comunidade ao redor do projeto e reter os clientes. Parece haver uma grande diferença entre os modelos de licenciamento livre e proprietário, já que o risco do fornecedor perder um cliente após a implantação é quase inexistente no atual cenário de mercado dominado pelo $P$-ERP, onde os atores globais ditam, na prática, as normas de mercado.

As diferenças entre o FOS-ERP e o P-ERP podem levar a uma mudança nas perspectivas relacionadas com o $P$-ERP, dominada pelo fornecedor, para perspectivas relacionadas ao FOS-ERP, mais direcionada ao cliente. Estas diferenças na condução da seleção, adoção e venda também trazem uma série de oportunidades e desafios para fornecedores e adotantes, os quais serão tratados nos tópicos seguintes. 

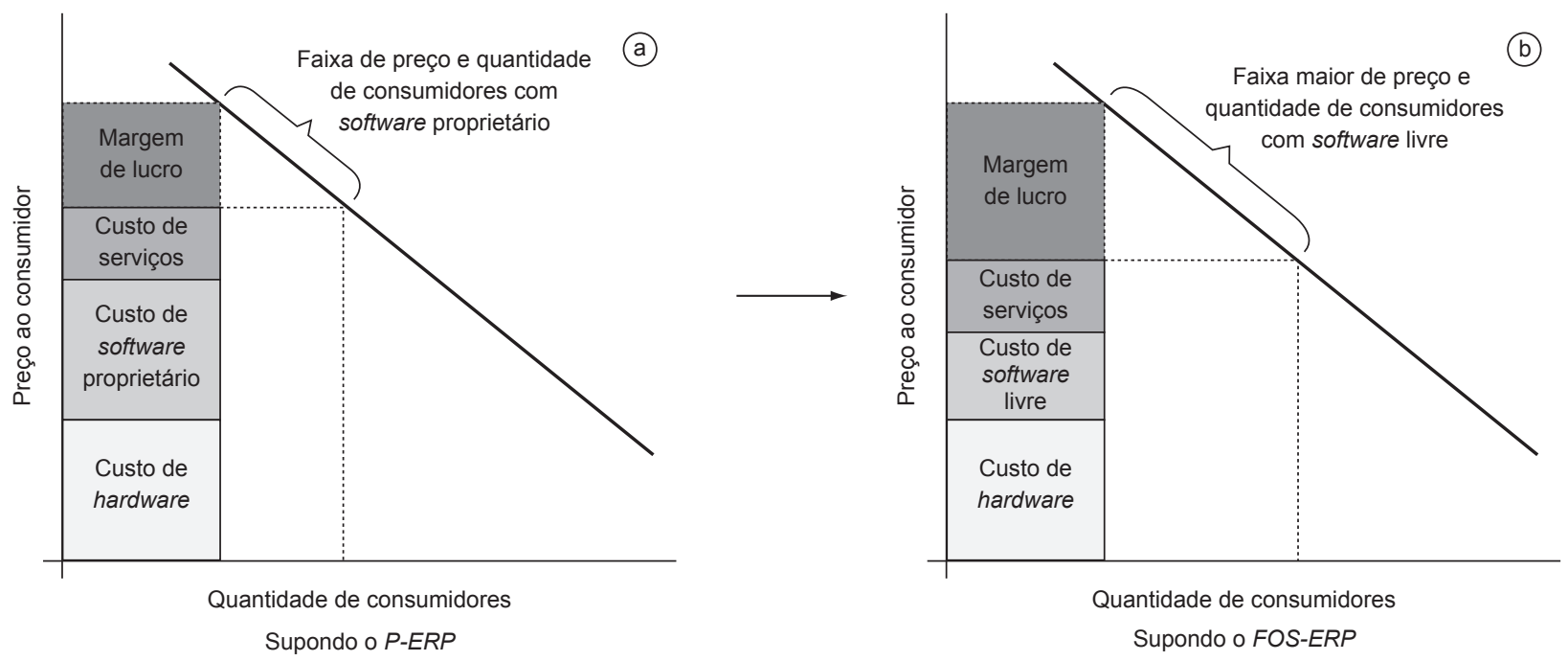

Figura 2. Faixas de preços e quantidade de clientes a) com o P-ERP e b) com o FOS-ERP. Fonte: Adaptado de Riehle, 2007.

\section{Oportunidades e desafios}

O FOS-ERP oferece uma série de oportunidades para atores que estão excluídos ou mal inseridos no mercado de $E R P$. Estas oportunidades são acompanhadas por uma série de desafios para as pequenas empresas de consultoria e as usuárias, como também para pesquisadores e colaboradores individuais.

\subsection{Para pequenas empresas de consultoria}

a) Oportunidades: os fornecedores de P-ERP impõem altos custos e um rígido conjunto de regras para empresas que desejam entrar em suas redes de parceria, aumentando as dificuldades para empresas pequenas que desejam se tornar atoras neste mercado. Em contraste, empresas menores de consultoria podem entrar no mercado de FOS-ERP de um modo incremental, aumentando seu compromisso com um projeto específico, à medida que novas oportunidades de negócio aparecem e trazem mais renda. Em outras palavras, as empresas podem começar a contribuir com pequenas melhorias no projeto como uma forma de adquirir conhecimento sobre a plataforma e estrutura do sistema. Conforme aparecem clientes para a solução, mais capital pode ser investido em um crescente comprometimento com o projeto. Além disso, com o aumento do investimento de risco na abertura de negócios com o FOSS, uma empresa menor pode até ser financiada de uma forma que seria muito improvável acontecer se ela trabalhasse também com uma solução de $P$-ERP, dada as restrições impostas pelos atores globais.

b) Desafios: se por um lado é mais fácil entrar no mercado, por outro é mais difícil reter clientes. O FOS-ERP fornece muito mais poder aos usuários, tornando-os mais exigentes. Manter o nível de qualidade entre uma rede heterogênea de provedores de serviços de consultoria é também um grande desafio. O FOS-ERP, em geral, apresenta poucos programas de certificação e garantia de qualidade que asseguram níveis de serviço aos clientes. Além disso, os céticos do FOS-ERP argumentam que poucas empresas de consultoria confiáveis possuem experiência na sua implementação. Mas são justamente tais programas que mantêm as empresas de consultoria menores longe do P-ERP, encaminhando-as ao FOS-ERP. Para uma empresa menor, uma possível solução para este impasse é começar com projetos menos ambiciosos e, então, seguir para os maiores, à medida que os processos de implantação e atividades relacionadas adquiram maturidade. Esta maturidade se tornará uma vantagem competitiva da empresa num mercado de FOS-ERP altamente competitivo.

\subsection{Para pequenos adotantes}

a) Oportunidades: custos menores abrem novas oportunidades para Pequenas e Médias Empresas (PME) adotarem e serem usuárias de ERP. Com a globalização, pequenas empresas sofrem mais e mais com a concorrência e, quando elas tentam modernizar seus processos, esbarram nos altos custos do $P$-ERP, ou precisam adotar soluções - também proprietárias - empacotadas, que são mais em conta, o que as liga a um único fornecedor que normalmente não possui uma rede de parceiros. Em contraste, o FOS-ERP apresenta custos menores e o suporte pode ser encontrado de diferentes formas, incluindo indivíduos e outras PME. Isto é verdadeiro também para governos e países em desenvolvimento, de modo geral. O FOS-ERP reduz os custos, ajudando governos a focar nos cidadãos e reduzir a dependência tecnológica dos atores globais. Na verdade, o FOSS em geral é uma oportunidade para os países em desenvolvimento 
mudarem de compradores para atores na indústria de software (OUÉDRAOGO, 2005).

b) Desafios: custos menores podem também significar que as PME e os governos tenham que lidar com níveis inferiores de serviço. Salienta-se, portanto, a necessidade de avaliar cuidadosamente o FOS-ERP e a maturidade de seus serviços de suporte. Adicionalmente, cabe citar a existência de barreiras na gestão de recursos de TI encontradas por este tipo de empresas, consequência de seu tamanho e recursos limitados, o que leva a uma estrutura de suporte de TI enxuta - quando não totalmente terceirizada, como acontece com as pequenas empresas De fato, Carvalho e Johansson (2009) destacam que no caso específico das pequenas empresas, a tendência é adotar o ERP no formato SaaS, delegando ao provedor toda a responsabilidade de prover o serviço, o que inclui o software de gestão.

\subsection{Para pesquisadores}

a) Oportunidades: possibilidade de compreender e participar de todo o processo que compõe uma solução de ERP, desde a concepção e desenvolvimento, passando pelos modelos de negócio, implantação, operação, manutenção e evolução do sistema. Como exemplo, os autores têm contribuído para um projeto de FOS-ERP (que desenvolve o ERP5, www.erp5.org) desde a sua concepção. Esta é realmente uma boa oportunidade, já que a maioria dos artigos de pesquisa sobre $E R P$ aborda assuntos relacionados à implantação e operação, considerando que as empresas de $P$-ERP geralmente não abrem seus projetos internos para os pesquisadores. Grupos menores de pesquisa podem encontrar seu caminho nesta área associando-se a um projeto de FOS-ERP, e contribuir em seu segmento de conhecimento específico.

b) Desafios: se, por um lado, a abertura do FOS-ERP pode fornecer aos pesquisadores informações sobre os processos de desenvolvimento e aspectos internos da solução, por outro é mais difícil obter informações de um conjunto de parceiros que, além de disperso, às vezes rege suas relações de maneira informal. Os aspectos sociais e econômicos, tais como as estruturas de remuneração, devem ser levados em consideração a fim de compreender a dinâmica do FOS-ERP, assim como em todo FOSS, trazendo mais componentes para serem analisados.

\subsection{Para colaboradores individuais}

a) Oportunidades: o FOS-ERP representa uma oportunidade única para os indivíduos instalarem uma estrutura de $E R P$ e compreenderem suas estruturas internas. Esta é uma chance de participar de um grande projeto de desenvolvimento de software sem ser funcionário de uma grande empresa (SPINELLIS, 2006). O desenvolvedor pode também adquirir conhecimento incremental do sistema e livremente obter suporte da comunidade, sem o investimento necessário nos programas de certificação e treinamentos de alto custo dos $P$-ERP. Desta forma, um indivíduo pode melhorar sua empregabilidade sem investir muitos recursos em treinamento, material de estudo e certificações. No futuro, essas vantagens podem fazer com que mais indivíduos entrem nas comunidades de FOS-ERP, atualmente formadas, em sua maior parte, por empresas, principalmente PME.

b) Desafios: aprender as estruturas internas de um FOSS, em geral significa empregar tempo considerável na compreesão da arquitetura do sistema e decisões de projeto. Além disso, o FOS-ERP - especialmente na atualidade - dispõe de pouca literatura para auxiliar no processo de aprendizagem e muitas vezes o indivíduo deve contar apenas com websites e fóruns de discussão e com a boa vontade dos membros da comunidade para adquirir conhecimentos mais profundos sobre a estrutura.

\section{Conclusões}

A crescente aceitação comercial dos FOS-ERP é um fato e seus custos menores, a sua adaptação mais fácil e o seu mercado fornecedor potencialmente mais competitivo podem forçar lentamente uma mudança no mercado de ERP a partir da perspectiva atual do fornecedor para uma perspectiva do cliente.

Este artigo apresentou particularidades, oportunidades e desafios relacionados aos FOS-ERP, contribuindo para um melhor entendimento de suas especificidades. É importante entender que, em geral, este tipo de software herda todas as vantagens e deficiências do software de código aberto em geral, além de mais algumas vantagens e deficiências específicas devido ao fato de serem sistemas empresariais.

O curto período de tempo que passou desde que o FOS-ERP apareceu no mercado e o número de usuários relativamente pequeno indica que a lista de oportunidades e desafios é um reflexo das atuais tendências, que devem ser confirmadas e melhor examinadas, à medida que ocorrem novas implantações. Em outras palavras, é necessário que mais dados sobre a implantação, customização, operação e evolução estejam disponíveis para que as tendências do FOS-ERP tornem-se fatos e números.

Assim, apesar do crescente interesse por este assunto, ainda existem muitos tópicos a serem explorados por pesquisadores e profissionais, como qualidade e confiabilidade do software, bem como novos modelos e estratégias de negócio, que podem surgir à medida que sua base de usuários aumenta. Portanto, na qualidade de assunto novo, a pesquisa em FOS-ERP possui um grande potencial devido às muitas perguntas que precisam ser respondidas, bem como devido às muitas tendências a serem confirmadas.

O meio acadêmico nacional deve interagir com demais interessados e tratar de questões que facilitem e viabilizem a utilização desses sistemas, resolvendo problemas específicos 
de nossas comunidades e empresas adotantes. Nesse sentido, em termos de estudos futuros, seria interessante prosseguir com a investigação de casos de seu emprego no Brasil. Especificamente, pretende-se investigar como o FOS-ERP pode colaborar para reduzir o problema de desalinhamento de requisitos (misfit), entre outras questões estratégicas e operacionais relacionadas ao seu desenvolvimento e à sua adoção.

\title{
An analysis of aspects related to the development and adoption of free open source Enterprise Resources Planning
}

\begin{abstract}
Enterprise Resources Planning (ERP) enables the processing of information required in an enterprise, using a single database. Much has been written about this type of software, addressing issues such as the high cost of licenses acquisitions, and the dependence on consultants for its adaptation and implementation in companies. Currently the development and use of ERP Free Open Source (FOS-ERP) has increased. However, this type of system is still not sufficiently explored, even in academia. This article analyses some papers on the subject, and raises questions that must be addressed by researchers and other stakeholders for the adequate use of these systems according to Brazil's reality. After an introduction to the subject, some differences between the FOS-ERP and its equivalent Proprietary ERP (P-ERP) are presented, in terms of business models, selection, customization and evolution. Next, some challenges and opportunities that FOS-ERP can offer to users, suppliers, researchers and individual contributors are described. This article expands the discussion about FOS-ERP, highlighting factors such as its potential for technological innovation and business strategy.
\end{abstract}

Keywords: Enterprise resources planning. ERP. Free software. Open source. Software development. Business strategy.

\section{Referências bibliográficas}

ALSHAWI, S.; THEMISTOCLEOUS, M.; ALMADANI, R. Integrating diverse ERP systems: a case study. The Journal of Enterprise Information Management, v. 17, n. 6, p. 454-462, 2004.

BOTTA-GENOULAZ, V.; MILLET, P. A.; GRABOT, B. A. Survey on the recent research literature on ERP systems. Computers in Industry, v. 56, n. 6, p. 510-522, 2005.

CAMPOS, R.; CARVALHO, R. A. Modeling architecture and reference models for the ERP5 project. In: IFIP INTERNATIONAL CONFERENCE ON RESEARCH AND PRACTICAL ISSUES OF ENTERPRISE INFORMATION SYSTEMS, 2006, Viena. Proceedings...

CARVALHO, R. A.; CAMPOS, R. A development process proposal for the ERP5 system. In: IEEE INTERNATIONAL CONFERENCE ON SYSTEMS, MAN, AND CYBERNETICS, 2006, Taipei. Proceedings...

CARVALHO, R. A.; COSTA, H. G.; XU, N. Risk based method for open source software adoption evaluation. In: CONGRESO LATINO-IBEROAMERICANO DE INVESTIGACIÓN OPERATIVA - CLAIO, 13, 2006, Montevideo. Proceedings... Asociación Latino-Iberoamericana de Investigación Operativa - ALIO, 2006.

CARVALHO, R. A. Issues on evaluating free/open source ERP systems. In: Research and practical issues of enterprise information systems. [S.1.]: Springer-Verlag, 2006. p. 667-676.

CARVALHO, R. A.; JOHANSSON, B. Enterprise resource planning systems for small and medium enterprises. In: RAMACHANDRAN,
M.; CARVALHO, R. A. (Org.). Handbook of research on software engineering and productivity technologies: Implications of globalisation. [S.1.]: IGI Global, 2009. p. 373-381.

CAULliRAUX, H. M.; PROENÇA, A.; PRADO, C. A. S. Enterprise resource planning systems from a strategic perspective. In: INTERNATIONAL CONFERENCE ON INDUSTRIAL ENGINEERING AND OPERATIONS MANAGEMENT, 6, 2000, Niteroi. Proceedings... ABEPRO, 2000.

CROWSTON, K.; HOWISON, J. Assessing the health of open source communities. IEEE Computer, v. 39, n. 5, p. 89-91, May 2006.

DREILING, A. et al. Open source enterprise systems: Towards a viable alternative. In: ANNUAL HAWAII INTERNATIONAL CONFERENCE ON SYSTEM SCIENCES, 38, 2005, Hawaii. Proceedings...

GALERA, R. G.; CAMPOS, R.; ROCHETTI, A. T. Uma análise comparativa entre construtores de linguagens de modelagem de empresas para o desenvolvimento de sistemas. In: SIMPÓSIO DE ENGENHARIA DE PRODUÇÃO - SIMPEP, 2008. Anais... Bauru: FEB/UNESP, 2008.

GONÇALVES, F. R.; PESSOA, M. C.; PRADO, J. P. Uma proposta de utilização de UML na implantação de sistemas ERP. In: CONGRESSO BRASILEIRO DE COMPUTAÇÃO. UFRGS, 2004. Disponível em: < www.niee.ufrgs.br/cbcomp/cbcomp2004>. Acesso em: 20 Março 2008. 
GOTH, G. Open source business models: ready for prime time. IEEE Software, v. 22, n. 6, p. 98-100, November/December 2005.

HERZOG, T. A comparison of open source ERP systems. Vienna, Austria, 2006. Thesis (Master) - Vienna University of Economics and Business Administration.

HONG, K.; KIM, Y. The critical success factors for ERP implementation: an organizational fit perspective. Information \& Management, v. 40, n. 1, p. 25-40, 2002.

IFIP/IFAC task force on architectures for enterprise integration. GERAM: Generalized enterprise reference architecture and methodology, 1999. 31 p.

JESUS, R. G.; OLIVEIRA, M. O. F. Implantação de sistemas ERP: tecnologia e pessoas na implantação do SAP R/3. Revista de Gestão da Tecnologia e Sistemas de Informação, v. 3, n. 3, p. 315-330, 2007.

KIM, H.; BOLDYREFF, C. Open Source ERP for SME. In: INTERNATIONAL CONFERENCE ON MANUFACTURING RESEARCH, 3, 2005, Cranfield, U.K. Proceedings...

KISSINGER, K. Can open source ERP software make an impact on the market? Novembro 2008. Disponível em: http://searchmanufacturingerp.techtarget.com/news/ article/0,289142,sid193_gci1339673,00.html. Acesso em: 13 Outubro 2009.

KOOCH, C. Open-Source ERP gains users. February, 2004. Disponível em: http://www.cio.com/archive/020104/tl_open. html. Acesso em: 15 Março 2008.

LECLAIRE, J. Open source, BI and ERP: The perfect match? Dezembro 2006. Disponível em: http://www.linuxinsider.com/ story/LjdZIB0x0j04cM/Open-Source-BI-and-ERP-The-PerfectMatch.xhtml. Acesso em: 15 Março 2008.

MENDES, J. V.; ESCRIVÃO FILHO, E. Atualização Tecnológica em pequenas e médias empresas: Proposta de roteiro para aquisição de sistemas integrados de gestão (ERP). Revista Gestão da Produção, v. 14, n. 2, p. 281-293, 2007.

MONNERAT, R. M.; CARVALHO, R. A.; CAMPOS, R. Enterprise systems modeling: the ERP5 development process. In: ACM SIMPOSIUM ON APPLIED COMPUTING, 23, 2008, Fortaleza. Anais...

OUÉDRAOGO, L. D. Policies of united nations system organizations towards the use of Open Source Software (OSS) in the secretariats. Geneva, 2005. 43 p.

RIEHLE, D. The Economic motivation of Open Source Software: stakeholder perspectives. IEEE Computer, v. 40, n. 4, p. 25-32, 2007.
ROCHETTI, A. T. Proposta de uma estrutura conceitual de modelagem de empresas para implementação do ERP5 baseada na ISO 19440:2007. Bauru, 2009. Dissertação (Mestrado em Engenharia e Produção) - Universidade Estadual Paulista - UNESP.

SERRANO, N.; SARRIERI, J. M. Open source ERPs: A new alternative for an old need. IEEE Software, v. 23, n. 3, p. 94-97, May-June 2006.

SILVA, C. M. F. et al. GERAM como arquitetura de referência para um ERP livre de código aberto . In: ENCONTRO NACIONAL DE ENGENHARIA DE PRODUÇÃO, 2006, Fortaleza. Anais... Rio de Janeiro: ABEPRO, 2006.

SILVA, F. P. C.; PEREIRA, N. A. Modelagem de processos de negócios na implementação de ERPs nacionais em PMEs. Revista de Produção, v. 16, n. 2, p. 341-352, 2006.

SMETS-SOLANES, J. P.; CARVALHO, R. A. ERP5: A nextgeneration, open-source ERP architecture. IEEE IT Professional, v. 5, n. 4, p. 38-44, 2003.

SPINELLIS, D. Open source and professional advancement. IEEE Software, v. 23, n. 5, p. 70-71, September/October 2006.

TIJUNELIS, P.; BARRELLA, W. D. Adaptação de ERPs. In: ENCONTRO NACIONAL DE ENGENHARIA DE PRODUÇÃO, 2003, Ouro Preto. Anais... Rio de Janeiro: ABEPRO, 2003.

WANG, F. R.; HE, D.; CHEN, J. Motivations of individuals and firms participating in open source communities. In: INTERNATIONAL CONFERENCE ON MACHINE LEARNING AND CYBERNETICS, 4, 2005. Proceedings...

WEST, J.; O'MAHONY, S. Contrasting community building in sponsored and community founded open source projects. In: ANNUAL HAWAII INTERNATIONAL CONFERENCE ON SYSTEM SCIENCES, 38, 2005, Hawai. Proceedings...

XU, N. An exploratory study of open source software based on public archives. Montreal, Canadá, 2003. Thesis (Master) - John Molson School of Business, Concordia University.

ZINNOV research and consulting. Penetration of open source in US enterprise software market - An overview. 2006. 37 p. Disponível em http://www.zinnov.com/presentation/Penetration_OSS_ enterprise_US_market.pdf. Acesso em: 12 Março 2008.

\section{Sobre os autores}

\section{Rogério Atem de Carvalho}

Instituto Federal Fluminense - IFF

R. Dr. Siqueira, 273, CEP 28030-130, Campos - RJ, Brasil

e-mail: ratem@iff.edu.br

\section{Renato de Campos}

Departamento de Engenharia de Produção - FEB, Universidade Estadual Paulista - UNESP Av. Eng. Luiz Edmundo C. Coube, 14-01, Vargem Limpa, CEP 17033-360, Bauru - SP, Brasil e-mail: rcampos@feb.unesp.br 